\title{
TRANSFORMACIONES EN EL MODELO DE GESTIŌN FEDERAL: UNA REFLEXIŌN DE LOS DESAFÍOS DEL FEDERALISMO COOPERATIVO A PARTIR DE LA EXPERIENCIA EN EL SECTOR EDUCATIVO ARGENTINO ${ }^{1}$
}

\author{
Horacio Cao (*) \\ Maximiliano Rey (*) \\ Universidad de Buenos Aires \\ Valeria A. Serafinoff $(\cdots)$ \\ Universidad Nacional de General Sarmiento
}

\section{RESUMEN}

Este trabajo analiza las transformaciones más recientes del federalismo argentino y los desafíos que se presentan a partir del proceso de migración de un modelo dual a otro cooperativo. Específicamente, reflexiona sobre los desafíos políticos e institucionales para la formulación e implementación de políticas públicas y las transformaciones producidas en términos de articulación de dichas políticas en contextos heterogéneos: grandes diferencias en los niveles de desarrollo regional, pero también en cuanto a la concepción político-ideológico y orientación de las políticas de las máximas autoridades del ejecutivo en cada nivel de gobierno. Se trabaja sobre los consejos federales,

considerados un instrumento que promueve la coordinación de políticas públicas entre el Estado nacional y los estados provinciales. Se explora en las transformaciones de la gestión federal de la política educativa puesto que aporta evidencia para identificar hitos significativos en el proceso de transformación de las modalidades de gestión del federalismo argentino y, en la experiencia reciente, como la consolidación del Consejo Federal de Educación (CFE) contribuye a mejorar las condiciones de articulación del modelo cooperativo. Se busca aportar al debate más instrumental sobre el federalismo y las relaciones intergubernamentales.

PALABRAS CLAVE :

federalismo, relaciones intergubernamentales, consejos federales, educación.

\footnotetext{
(•)E-mail: horaciocao33@hotmail.com

$(\cdot \bullet)$ E-mail: maxirey@hotmail.com

(‥)E-mail: valeria.serafinoff@gmail.com
}

RECEPCIÓN: 24/03/16

ACEPTACIÓN FINAL: 12/10/16 